\title{
3D MODELING AND NUMERICAL SIMULATION OF A WATER SOFTENER DEVICE
}

\author{
Dorin EFTIMIE \\ "Dunarea de Jos" University of Galati, Romania \\ e-mail: dorin.eftimie@ugal.ro
}

\begin{abstract}
Water treatment in the magnetic field is not an officially recognized method of water softening. This method can be promoted as an alternative to water softening without potentially toxic chemical agents.

The water softener device consisting of 3 pairs of permanent magnets made of neodymium was designed with the help of Solid Edge software.

Numerical simulation was performed using ANSYS WORKBENCH finite element analysis software using the FluidFlow (CFX) module. With this analysis module the dynamic analysis of the fluid flow through a pipe was performed.

Three numerical analyses of the water flow through a pipe with an internal diameter of $20 \mathrm{~mm}$ were performed at a pressure of 2 bars, namely:

- the water flow without magnetic influences;

- the water flow under the magnetic influence of the softener device positioned longitudinally with the pipe;

- the water flow under the magnetic influence of the softener device positioned transversely with the pipe.

The conclusions of these simulations can be the basis for future research in order to optimize this type of device.
\end{abstract}

KEYWORDS: magnetic water treatment analyse, water softening, water softener device

\section{Overview}

Water has an essential role in life maintenance. Life could not exist without water. Every organism has water in its composition, all organs, tissues or biological fluids. Water dissolves and transports assimilated and unassimilated substances. Water keeps constant salts concentration in the body. Water takes part in temperature adjustment, evaporating on the surface of the body. In plants, water contributes to osmotic phenomena and has a great importance in the photosynthesis process.

Water has the ability to dissolve very many substances. In nature, water is not chemically pure. Natural waters contain varying amounts of substances, from gases and dissolved salts to organic substances and bacteria. Water is a vital part in many metabolic processes of the body. It is a universal solvent of living matter, being the molecular framework in which life processes are carried out.

Drinking water is that water whose quality must ensure perfect health of the end user. Coming from the natural environment, water must undergo a series of treatments that make it suitable for human consumption.

Magnetic treatment - even if this method of water treatment for softening is not officially recognized, water treatment in the magnetic field is promoted as a safe alternative of treating water with potentially toxic chemical agents.

Water treatment systems with magnetic devices are an alternative to conventional chemical or physical treatment systems and have the advantage of removing mineral and organic suspensions such as algae or microorganisms, inhibiting corrosion of pipes used for water transport and so on. When a stream of water is subjected to a magnetic field, which can be induced by an electric field or by the media of minerals, such as magnetite, polarization of the constituent minerals of the water occurs and the ionic charge changes of these minerals. The effluent of such an installation is less hard water, forming groups (the classical groups of cells of 12-18 molecules of water are divided into 6 groups, following ionization) with high hydration properties 
and with high penetration capabilities in the body (Fig. 1) [1].

Many such devices consist of one or more permanent magnets applied, either inside or on the outer surface of the water inlet pipe. The water is exposed to the magnetic field as it flows through the pipe between the magnets (Fig. 2) [2]. Water and water solutions passed through these magnetic fields acquire finer and more homogeneous structures. This increases the fluidity of the water and contributes to increasing the ability of the water to dissolve various constituents, such as minerals and vitamins), and therefore improves the biological activity of the solutions, which positively affects the performance of humans, animals and plants.
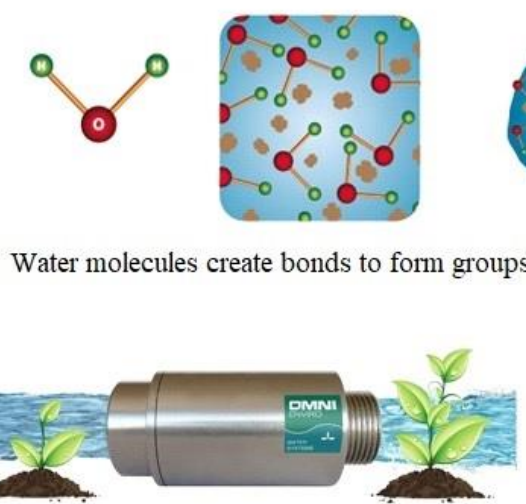

Magnetic devices
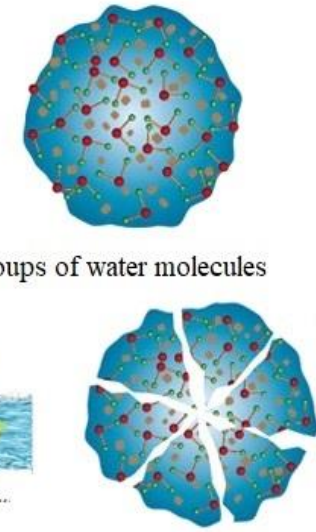

separate the groups

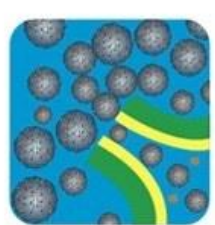

Many of the groups are too large to enter the cell

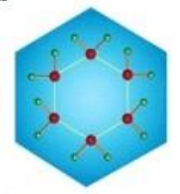

in smaller

hexagonal groups

Fig. 1. The principle of magnetic water

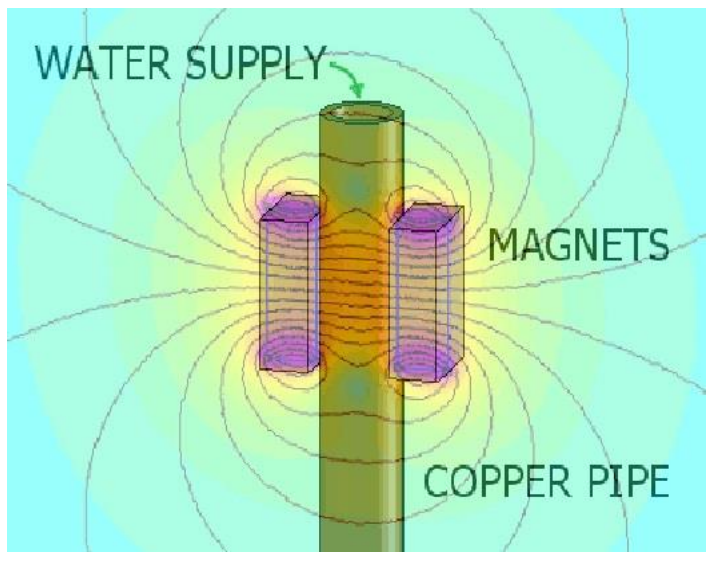

Fig. 2. Magnetic treatment assembly

Magnets affect the angle of connection between the hydrogen and the oxygen atom in the water molecule. The magnetization of water causes the hydrogen-oxygen bond angle in the water molecule to decrease from 104 to 103 degrees. This, in turn, causes the water molecule to gather together in groups of 6-7 molecules and not 10-12 or more (Fig. 3) [1]. Smaller groups lead to better water absorption through cell walls. Dr. Michael Lam, in his study of magnetized water, reports that there were numerous mentions of people who drank magnetized water every day who were cured of many chronic degenerative diseases, such as kidney stones and arthritis.
The theory of water magnetization is that water flows underground, comes into contact with the electromagnetic field of the earth and the magnetic charge is passed into the water. When the water is treated for drinking, it is passed through metal pipes, which demagnetize it. Magnetizing the drinking water breaks the surface tension, making it easier to absorb from every cell in the body. In addition, there is a strong side benefit. Applying a magnetic field to water can increase its $\mathrm{pH}$ (up to a whole point, depending on the water).

Magnetically treated water directs water through a strong magnetic field. By placing two strong neodymium magnets on each side of the inlet pipe, all the water passes through a strong, uniform magnetic field. Treating the water with magnets does not remove calcium from the water, but claims that it changes the structure of the deposits that are formed, causing them to adhere less to the surfaces.

A large variety of magnetic water treatment devices are available, but most consist of one or more permanent magnets fixed inside or on the outer surface of the water inlet pipe. The water is exposed to the magnetic field as it flows through the pipe between the magnets (Fig. 2). An alternative approach is to use the electrical current that runs through wire strands wrapped around the water pipe to generate the magnetic field.

Suppliers of magnetic water treatment devices claim that exposing water to a magnetic field will 
reduce the "effective" water hardness. Typical claims include the removal of limestone deposits, lower water heating bills, longer service life of water heating systems and appliances, as well as more efficient use of soaps and detergents. Thus, it is claimed that magnetic water treatment offers all the benefits of ion exchange softening.

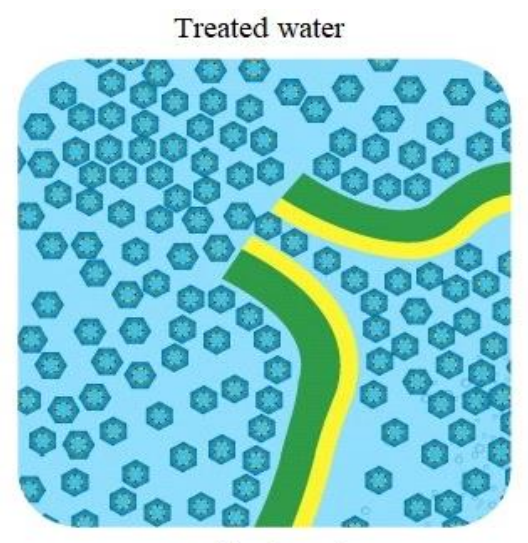

Total hydratation

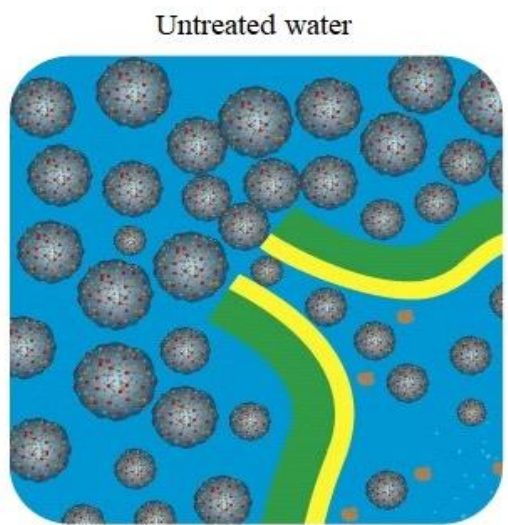

Partial hydratation

Fig. 3. Water absorption in cells

The "effective" hardness is claimed to be reduced by magnetic treatment. Magnesium or calcium is not removed from magnetically treated water. Instead, the claim is that the magnetic field decreases the tendency of dissolved minerals to form limestone. Even though the concentration of dissolved minerals indicates that it is still hard, the magnetically treated water is assumed to behave as softened water.

The main objectives of this paper are to demonstrate the following issues:

- reduction of the surface tension and internal water tension;

- increasing the ability of water to dissolve various substances, such as minerals from the soil;

- improving the biological activity of the solutions that positively affects the performance of the plants; generated by a water sample passed through a device for water magnetizing.

In the first phase, it is necessary to construct a device for water magnetization consisting of two metal frameworks, each one consisting of three neodymium magnets.

The device for water magnetizing has the role of magnetizing and purifying the water that passes through the magnetic field created by this device.

Practical determinations of the two samples of non-magnetized and magnetized water are performed for:

- comparison of the parameters values analysed in non-magnetized and magnetized water and evaluation of the obtained results

- evaluation of the toxic ion concentrations, respectively the elements: phenols, detergents, chloride, sulphate, boron, molybdenum, lead, cadmium, total chromium. Sometimes, even if the level of salt (chlorides) is not excessive, one or more of these elements can become toxic to plants. Many plants are particularly sensitive to boron.

Following the bibliographic studies performed, it was designed a device consisting of two metallic frameworks that have three permanent neodymium magnets each other (Tab. 1, Fig. 4).

Table 1. Magnets properties

\begin{tabular}{|c|c|}
\hline Material & NdFeB \\
\hline Form & Block \\
\hline Dimensions & $60 \times 30 \times 15 \mathrm{~mm}$ \\
\hline Tolerance & $+/-0.1 \mathrm{~mm}$ \\
\hline $\begin{array}{c}\text { Nickel-plated } \\
\text { coating }\end{array}$ & $\begin{array}{c}\text { Nickel-plated } \\
(\mathrm{Ni}-\mathrm{Cu}-\mathrm{Ni})\end{array}$ \\
\hline Magnetization & $\mathrm{N} 40$ \\
\hline Attraction force & $\mathrm{approx} .56 \mathrm{~kg}$ \\
\hline $\begin{array}{c}\text { Maximum working } \\
\text { temperature }\end{array}$ & $80{ }^{\circ} \mathrm{C}$ \\
\hline Weight & $210 \mathrm{~g}$ \\
\hline
\end{tabular}

Neodymium magnets are composed of iron, boron and neodymium, being ten times stronger than traditional magnets (ferrite magnets), known as the most powerful and advanced magnets in the world. Neodymium magnets are generally covered by a triple layer of Nickel-Copper-Nickel or epoxy resin offering durability and corrosion protection. 


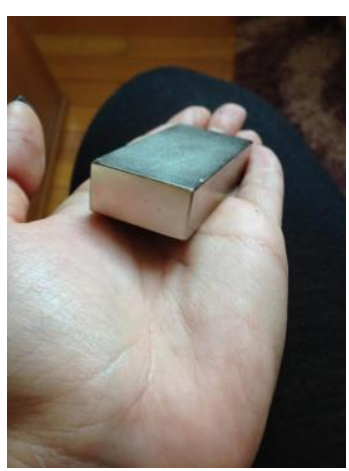

Fig. 4. Permanent magnet

The modelling of the mechanism for water magnetization was designed using the Solid Edge software.

The 3D modelling was performed also with the device positioned longitudinally (Fig. 5) and transversely (Fig. 6) in order to make a comparative analysis of the effects resulted by changing the orientation of the magnetic field.

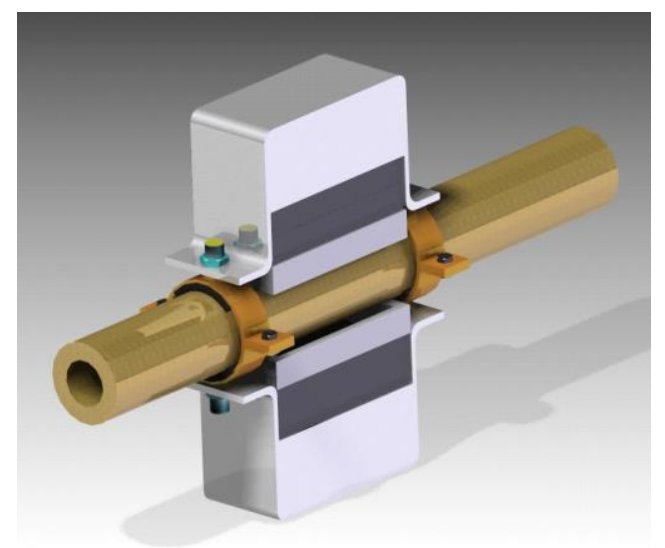

Fig. 5. 3D modelling of the device positioned longitudinally on the pipe

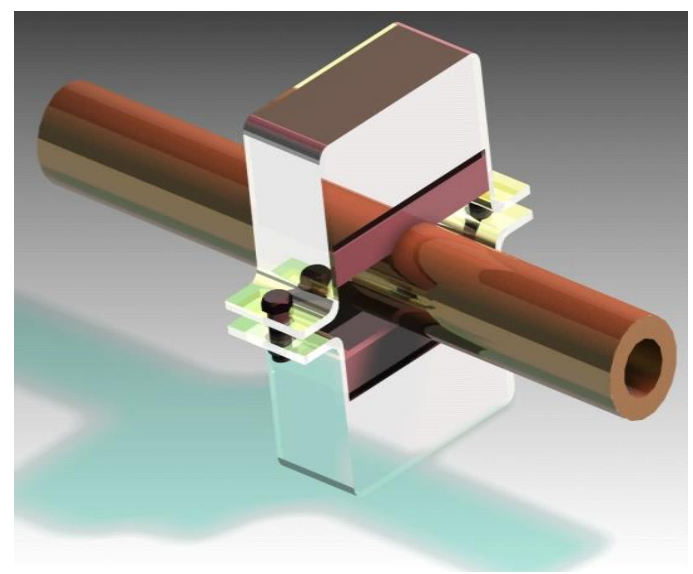

Fig. 6. 3D modelling of the device positioned transversally on the pipe

\section{Numerical simulation of the device for water magnetization}

The device for water magnetizing has the role of magnetizing and purifying the water that passes through the magnetic field created by it. To demonstrate how water magnetization is performed and the effect of the magnetization device on water, a numerical simulation was performed using the ANSYS WORKBENCH finite element analysis software using the Fluid Flow (CFX) module [3]. With the help of this analysis module, dynamic analysis of fluid flow through a pipe was performed.

Three numerical analyses of water flow through a pipe were performed, namely:

- the water flow without magnetic influences;

- the water flow under the magnetic influence of the device for water magnetizing positioned longitudinally with the pipe;

- the water flow under the magnetic influence of the device for water magnetizing transversally positioned with the pipe.

It was considered that the water flows through a pipe with an internal diameter of $20 \mathrm{~mm}$. The limit condition that has been applied for the water flow through the pipe is the pressure of 2 bars that is also in the current water supply systems of the houses.

The limit condition can also be seen in Figure 7.

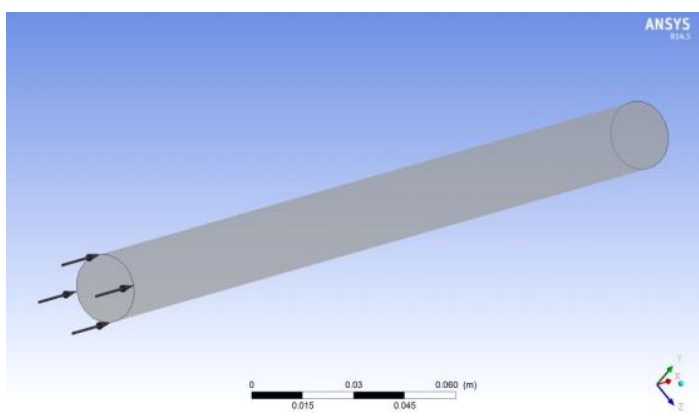

Fig. 7. Limit conditions at the water flow through the pipe

Following the analysis, a quasi-laminar flow was obtained.

The analysis was performed using the Fluid Flow (CFX) module in which an electrohydrodynamic analysis was performed. The limit conditions imposed in this analysis were primarily the flow conditions of the fluid through the pipe under the pressure of 2 bars and on the other hand the magnetic intensity of the neodymium magnets inside the device.

Following the analysis, a turbulent flow was obtained due to the very strong magnetic forces acting on the flow of the fluid. The current lines resulting from this analysis can be seen in Figure 8. 


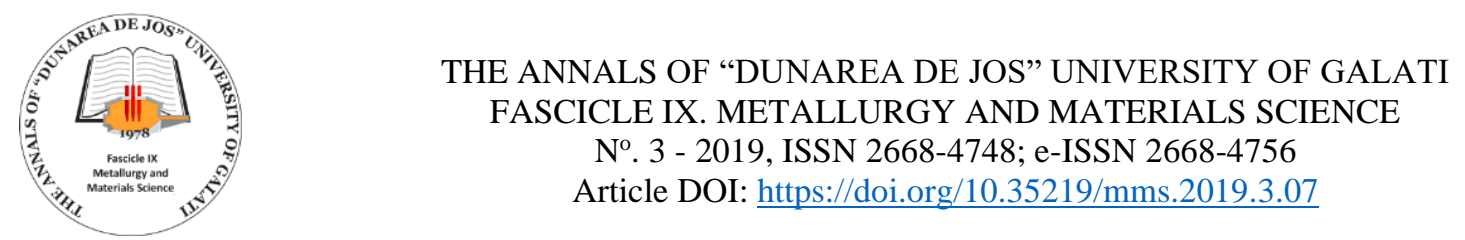

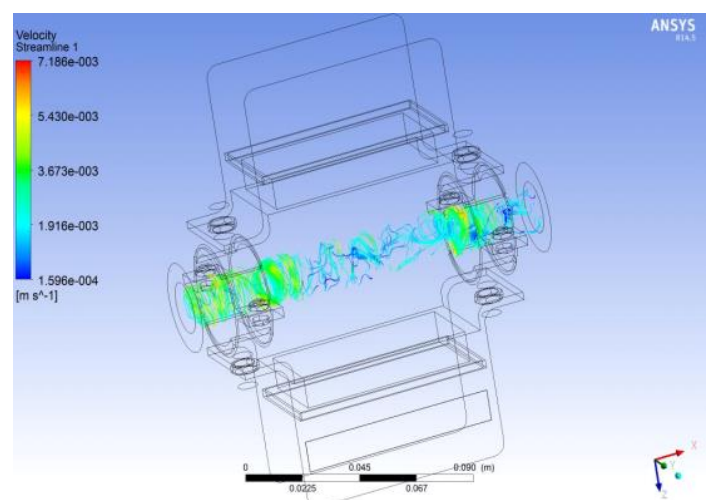

Fig. 8. Lines of fluid flow through the pipe

In Figure 10 can be seen how the velocity of the fluid is also influenced by the magnetic forces. In the area of magnets there is a slowdown of the fluid. This can be seen in Figure 9, how the fluid velocity at the entrance is at maximum level, highlighted by the red colour, and then decreases next to the magnets.

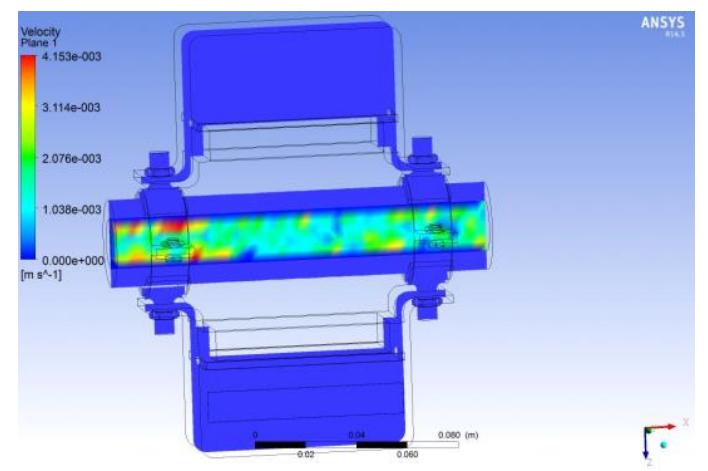

Fig. 9. Distribution of flow velocities

Figure 10 shows how water is magnetized with a magnetic intensity of $0.0002 \mathrm{~T}$. As shown in this figure, the maximum magnetic intensity is in the center of the magnets, where the magnetic forces are also at maximum value.

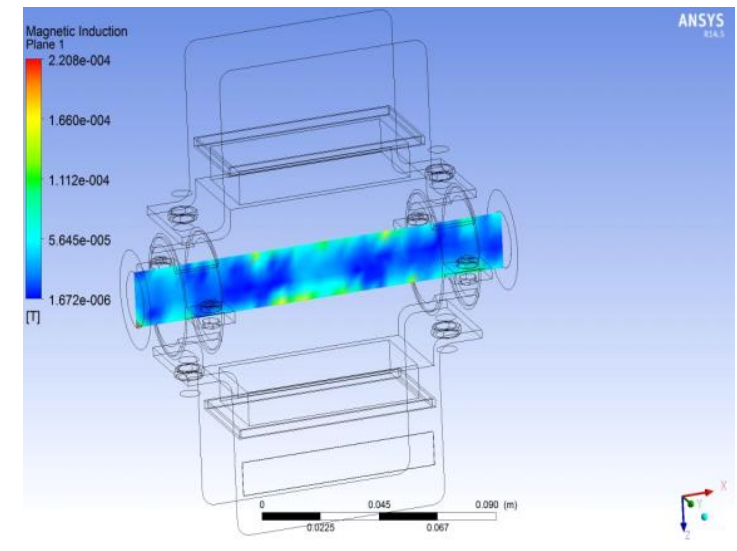

Fig. 10. Magnetization of water
Due to the velocity and magnetic influences the water is charged energetically with current density of $7 * 10^{-9} \mathrm{~A} / \mathrm{m}^{2}$. In Figure 11 you can see the energetic distribution of water. As can be seen the water is charged energetically.

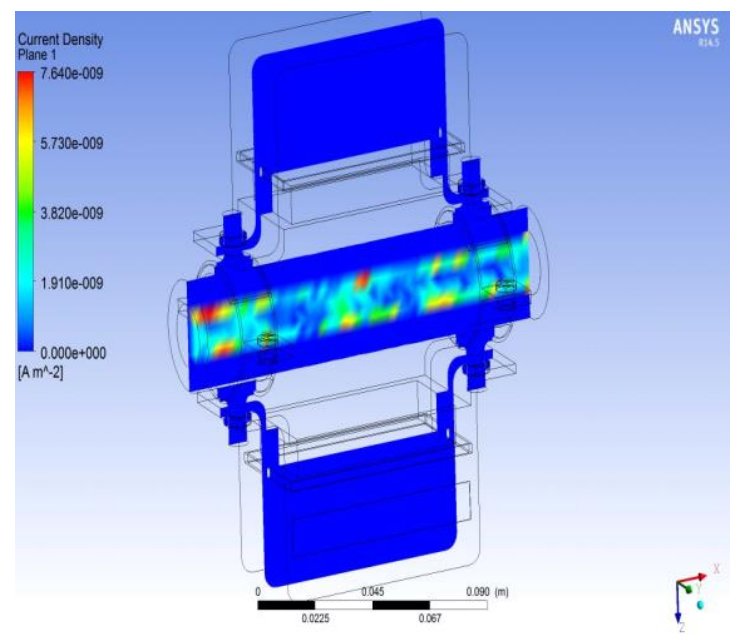

Fig. 11. Energetic distribution of water

In the case of the device which is transversally positioned, see Figure 12, the same limit conditions were applied as in the case of longitudinal positioning. These two analyzes that were performed, aimed at observing the efficiency of the device according to its positioning on the pipe.

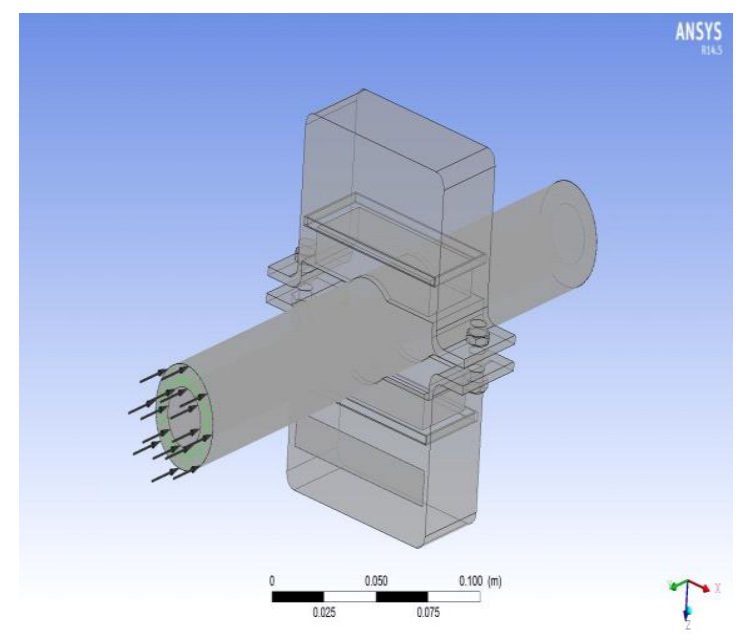

Fig. 12. The limit conditions for the transversally positioned device

Following the analysis, results were obtained for the flow lines, the velocities distribution, the magnetic distribution and the energetic distribution.

In Figure 13 it can be seen the flow lines in case of flow under the magnetic influence of the device positioned transversally with the pipe. As it can be 
seen, the flow is turbulent having the maximum turbulence in the central area of the magnets where the maximum forces of magnetic influence are concentrated.

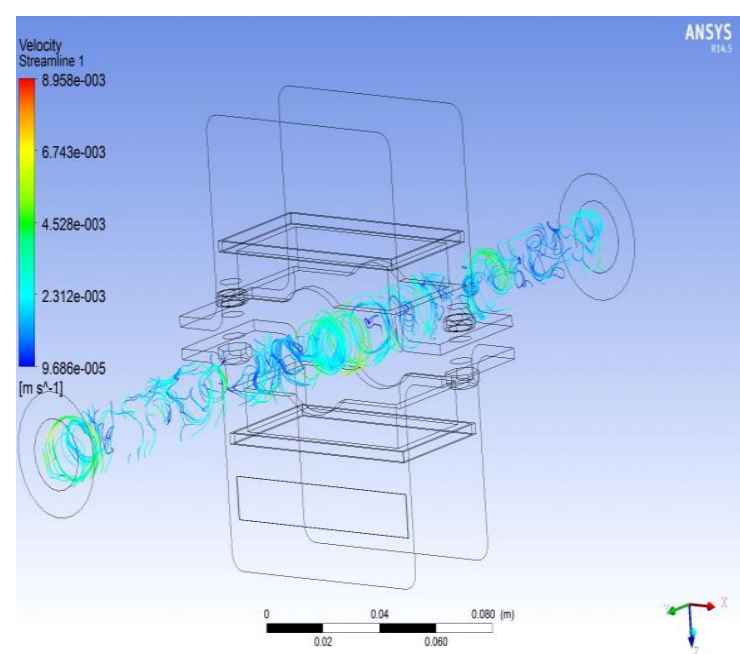

Fig. 13. Flow lines thru pipe with transversally positioned device

In Figure 14 can be seen a distribution of the fluid flow velocities. In this case the fluid has a higher velocity than in the first case because the area of magnetic influence is smaller than in the first case, and the magnetic forces are concentrated on a smaller portion of the pipe.

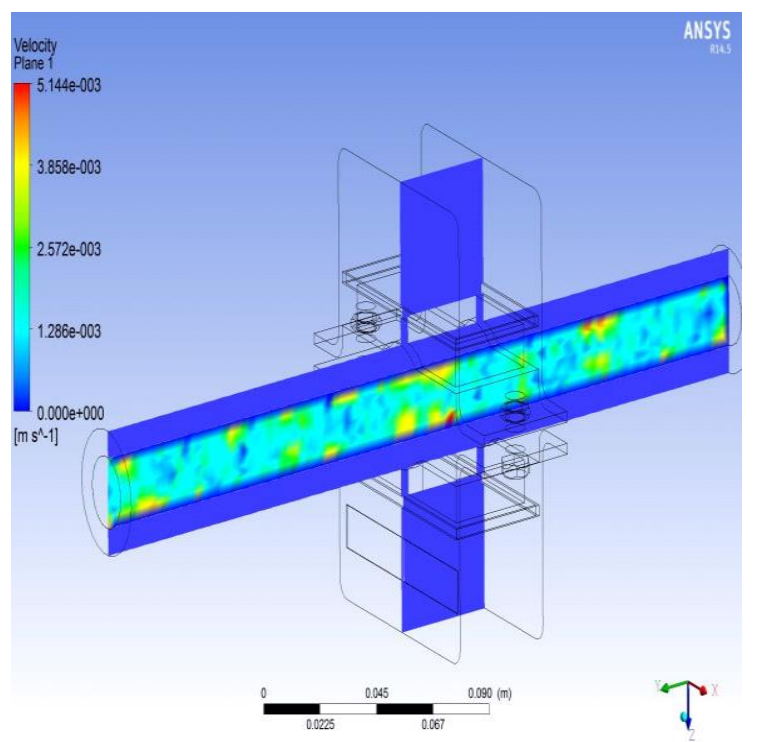

Fig. 14. Distribution of flow velocity for the case of the transversally positioned device

Figure 15 also shows the water magnetization distribution. In this case, the magnetic intensity of the water is lower than in the first case. The magnetization of water is twice smaller as in the case with the device positioned longitudinally along the pipe.

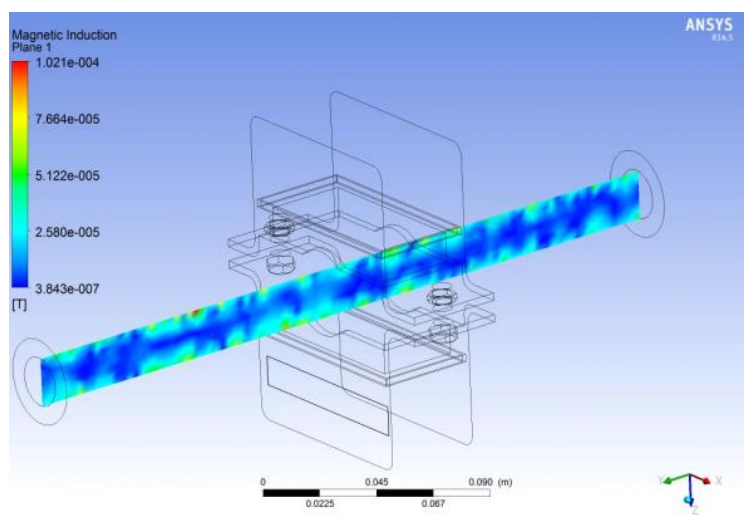

Fig. 15. Magnetization of water with the transversally positioned device

Lowering the magnetization of the water increases the density of the flow created because the magnetic intensity is concentrated in a smaller area than in the first case. The maximum flow density is $8 \times 10^{-9} \mathrm{~A} / \mathrm{m}^{2}$. The energetic distribution of water can be seen in Figure 16.

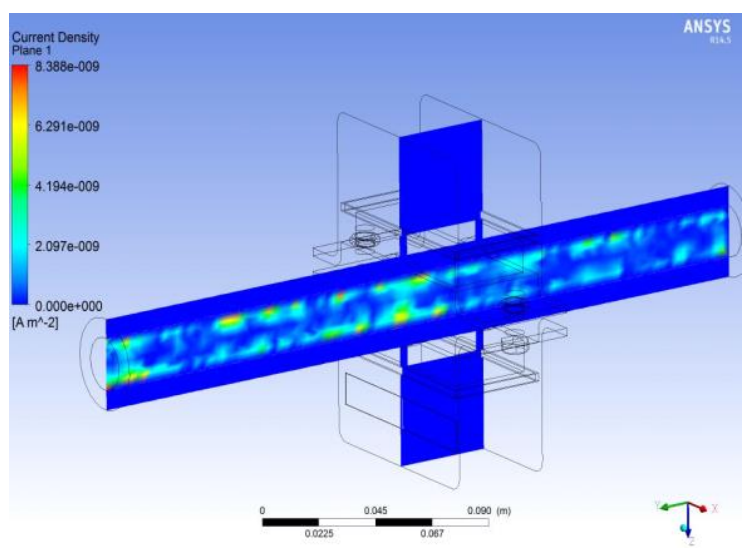

Fig. 16. Energetic distribution of water

\section{Conclusions}

Following the study there were found the following:

- the laminar flow of water is transformed into a turbulent flow under the strong influence of magnetic forces;

- the water under the strong influence of the magnetic field is magnetized;

- the flow of water through a strong magnetic field electrifies the water;

- for maximum efficiency, the proposed device for water magnetization must be positioned 
THE ANNALS OF "DUNAREA DE JOS" UNIVERSITY OF GALATI

FASCICLE IX. METALLURGY AND MATERIALS SCIENCE

№. 3 - 2019, ISSN 2668-4748; e-ISSN 2668-4756

Article DOI: https://doi.org/10.35219/mms.2019.3.07

longitudinally on the pipe through which the water flows.

Following the numerical simulation, the variant of the device positioned longitudinally on the pipe was chosen for the construction.

\section{References}

[1]. ***, http://www.omnienviro.com/magnetized-water/.

[2]. ***, https://www.kjmagnetics.com/blog.aspp=water-treatment.

[3]. ***, https://www.ansys.com/products/fluids/ansys-cfx. 